\title{
Atribuições do enfermeiro no centro cirúrgico: percepções sobre si no cenário intraoperatório
}

\author{
Roles of nurses in the operating room: perceptions of themselves in the intraoperative setting \\ Funciones de las enfermeras en la sala de operaciones: las percepciones de sí mismos en el \\ intraoperatorio
}

Recebido: 11/08/2021 | Revisado: 17/08/2021 | Aceito: 23/08/2021 | Publicado: 24/08/2021

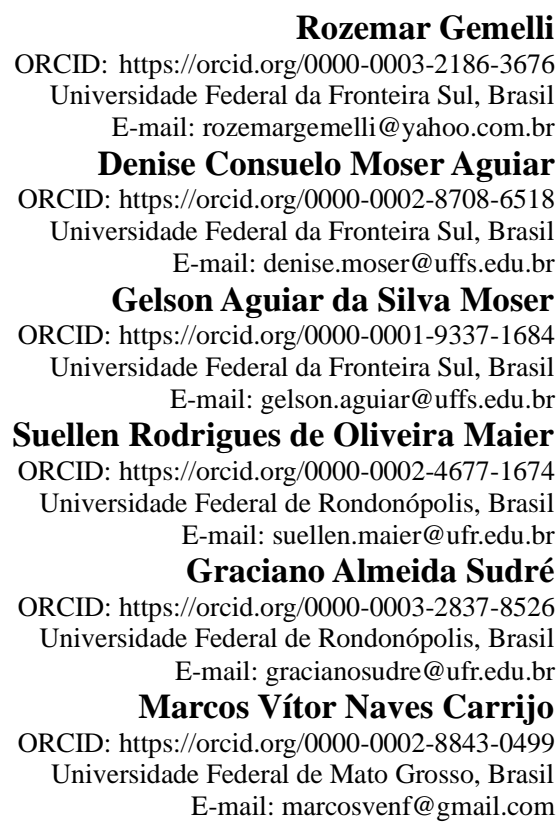

\begin{abstract}
Resumo
Objetivo: Compreender a percepção do enfermeiro, atuante no centro cirúrgico, com relação à assistência ofertada ao paciente durante o transoperatório em um hospital do oeste de Santa Catarina. Metodologia: Trata-se de uma pesquisa qualitativa, demarcado com pressupostos do estudo etnográfico, a partir de entrevistas e observação participante, que envolveu um grupo de enfermeiros atuantes no centro cirúrgico de um hospital situado na região oeste de Santa Catarina. Foram incluídos enfermeiros atuantes no setor por um período superior a seis meses, não havendo nenhum critério de exclusão. A pesquisa teve aprovação do comitê de ética em pesquisa a partir do parecer consubstanciado $\mathrm{n}^{\circ}$ 3.130.468. Resultados: Três categorias emergiram do processo de análise: "O cuidado durante a experiência cirúrgica: enfermeira em ação", "Cuidado Perioperatório: dispositivos possíveis no cotidiano" e "Percepção dos Enfermeiros frente ao cuidado no centro cirúrgico". Conclusão: Destaca-se a sobrecarga de trabalho ao qual toda a equipe de enfermagem está exposta, sendo esse o principal fator que restringe a atuação do enfermeiro junto ao paciente na oferta do cuidado, situação que evidencia a necessidade do enfermeiro ou enfermeira coordenador e de outro(a) assistencial, ambos com atuação direta na oferta do cuidado ao paciente junto à equipe, almejando um trabalho interprofissional.
\end{abstract}

Palavras-chave: Enfermeiros; Enfermagem perioperatória; Cuidados de enfermagem; Centros cirúrgicos.

\begin{abstract}
Objective: To understand the perception of nurses working in the operating room regarding the care offered to patients during the transoperative period in a hospital in the west of Santa Catarina. Methodology: This is a qualitative research, demarcated with assumptions of the ethnographic study, based on interviews and participant observation, which involved a group of nurses working in the operating room of a hospital located in the western region of Santa Catarina. Nurses working in the sector for a period longer than six months were included, with no exclusion criteria. The research was approved by the research ethics committee based on the embodied opinion $\mathrm{n}^{\circ}$ 3.130.468. Results: Three categories emerged from the analysis process: "Care during the surgical experience: nurses in action", "Perioperative care: possible devices in everyday life" and "Nurses' perception regarding care in the operating room".
\end{abstract}


Conclusion: The work overload to which the entire nursing team is exposed is highlighted, which is the main factor that restricts the nurse's work with the patient in the provision of care, a situation that highlights the need for the nurse or nurse coordinator and another care, both with direct action in providing care to the patient with the team, aiming for an interprofessional work.

Keywords: Nurses; Perioperative nursing; Nursing care; Surgical centers.

\section{Resumen}

Objetivo: Comprender la percepción de los enfermeros que laboran en el quirófano sobre la atención brindada a los pacientes durante el transoperatorio en un hospital del occidente de Santa Catarina. Metodología: Se trata de una investigación cualitativa, demarcada con supuestos del estudio etnográfico, a partir de entrevistas y observación participante, que involucró a un grupo de enfermeras que laboraban en el quirófano de un hospital ubicado en la región occidental de Santa Catarina. Se incluyeron enfermeras que laboran en el sector por un período superior a seis meses, sin criterios de exclusión. La investigación fue aprobada por el comité de ética en investigación con base en el dictamen incorporado $n^{\circ} 3.130 .468$. Resultados: Del proceso de análisis surgieron tres categorías: "Atención durante la experiencia quirúrgica: enfermeras en acción", "Atención perioperatoria: posibles dispositivos en la vida cotidiana" y "Percepción de las enfermeras sobre el cuidado en quirófano". Conclusión: Se destaca la sobrecarga de trabajo a la que está expuesto todo el equipo de enfermería, que es el principal factor que restringe el trabajo de la enfermera con el paciente en la prestación de cuidados, situación que resalta la necesidad de la enfermera o enfermera coordinadora y otro cuidado, ambos con acción directa en la atención al paciente con el equipo, buscando un trabajo interprofesional.

Palabras clave: Enfermeros; Enfermería perioperatoria; Cuidado de enfermera; Centros quirúrgicos.

\section{Introdução}

Para um ambiente cirúrgico seguro, as ações de enfermagem devem abranger cuidados físicos e psicossociais com início no período pré-operatório, permear pela compreensão das fases e intervenções transoperatórias e pela identificação das possíveis necessidades de saúde que emergem no pós-operatório (Jonhstone, 2020).

Historicamente a evolução das práticas cirúrgicas articulam-se ao trabalho da enfermagem, inicialmente resumindo-se a contenção da pessoa e garantia de ambientes limpos, atualmente envolvem muito mais que isso, com desenvolvimento de competências importantes e de aspecto técnico científico executado de maneira critico reflexiva (Santos et al, 2018), permeando o domínio de habilidades cognitivas, psicomotoras e atitudinais.

Nesse sentido, dentre as diferentes atribuições, é papel do enfermeiro e da enfermeira garantir a segurança do paciente; compreender o efeito de suas intervenções; comunicar-se com clareza com a pessoa submetida ao procedimento, família, cuidadores e equipe1, além disso, exercer a função assistencial e de coordenação da unidade (Sobecc, 2017).

A participação desse profissional no centro cirúrgico começou devido a necessidade de trabalhadores para suprir algumas necessidades específicas relacionadas aos procedimentos, cuidados, gestão e provisão de materiais (Silva et al, 2017) e, assim como para elaboração, implementação, controle e avaliação de normas e rotinas específicas (Klein et al., 2018).

Atualmente, percebe-se um afastamento do enfermeiro da assistência direta a pessoa, e uma aproximação das questões burocráticas, uma vez que as ações administrativas são intensas nas unidades de centro cirúrgico. As atribuições gerenciais são tão relevantes quanto as relacionadas ao cuidado direto e repercutem na atenção à pessoa no atendimento às suas necessidades de saúde, com isso, é imperativo que o enfermeiro pondere e gerencie seu tempo com a finalidade de desempenhar as duas funções (Koch et al, 2018).

Contudo, as funções relacionadas ao cuidar no contexto perioperatório atribuídas ao enfermeiro, por vezes não são realizadas devido às tarefas voltadas à gestão do trabalho no setor. Dessa forma, emerge o interrogar: como o enfermeiro compreende seu trabalho assistencial no centro cirúrgico?

Nesta perspectiva, essa pesquisa foi desenvolvida com o objetivo de compreender qual a percepção do enfermeiro, atuante no centro cirúrgico, com relação à assistência ofertada ao paciente durante o transoperatório em um hospital do oeste de Santa Catarina.

A pesquisa apresenta uma contribuição ao trabalho em saúde, a partir do momento em que é propiciado um espaço de 
fala ao profissional, sua percepção em relação ao objeto é modificada e surge uma oportunidade para repensar a prática profissional.

\section{Metodologia}

Trata-se de uma pesquisa descritiva, com técnicas próprias das investigações qualitativas. Essa abordagem permite que a imaginação e a criatividade levem os pesquisadores a propor trabalhos que explorem novos enfoques, além disso, a pesquisa qualitativa requer atitudes fundamentais, que possibilitam o aprofundamento do sentido das ações, aspecto essencial para o enfoque teórico adotado (Minayo \& Costa 2018).

Em relação à abordagem teórica, iniciou-se com a intenção de pesquisa, atitude do pesquisador e se efetivou com a verdadeira inserção no campo, para o desenvolvimento e tessitura de experiências etnográficas, cabe destacar que permeou atitudes do pesquisador de descrição densa do cenário observado, atenção às informações e encontros completamente transformados por processos reflexivos e compartilhamento com o grupo de pesquisa.

O presente estudo foi realizado em um hospital brasileiro, envolveu quatro enfermeiros, de diferentes turnos de trabalho inseridos no centro cirúrgico, para distinguir as entrevistas os participantes serão nomeados de ENF1, ENF2, ENF3 e ENF4. Foram incluídos enfermeiros atuantes no setor por um período superior a seis meses, não havendo nenhum critério de exclusão.

A entrada no campo, coleta e registro das informações ocorreu no primeiro semestre do ano de 2019, inicialmente com uso de roteiro semiestruturado para entrevista. As mesmas ocorreram em sala privada do centro cirúrgico, ambiente que garantiu a não interrupção, respeitando-se a disponibilidade dos enfermeiros envolvidos - essa modalidade obedeceu a uma ferramenta guia para facilitar a interlocução com os participantes do estudo (Minayo \& Costa 2018).

Ocorreu também, em um segundo momento, de forma mais intensa e abrangente, observação participante. O pesquisador adentrou ao campo nos períodos diurno e noturno, ação que se demonstrou indispensável no processo de produção dos dados. Essa imersão viabilizou a escrita em diário de campo, matéria prima do enfoque etnográfico, que permitiu a convivência efetiva com os participantes em seu cotidiano de trabalho e registro atento do que esses encontros produziram no pesquisador.

Este estudo faz parte de um projeto guarda-chuva intitulado "Corpo e Corporeidade no Cotidiano do Centro Cirúrgico: bordando o cuidado e a formação no labirinto da equipe de enfermagem", o qual recebeu anuência do comitê de ética em pesquisa da Universidade Federal da Fronteira Sul (UFFS), sob parecer 3.130.487 e Certificação de Apresentação e Apreciação Ética (CAAE): 03509918.3.0000.5564, conforme disposto na Resolução no 466/2012. O tratamento dos dados se deu por meio da análise de conteúdo, seguindo as respectivas etapas: a) organização da análise; b) codificação; c) categorização; d) tratamento dos resultados, inferência e a interpretação dos resultados (Bardin, 2016).

\section{Resultados}

O processo de construção das etapas que representa o resultado foi detalhado nos quadros organizados por meio da composição das informações coletadas e observadas durante o movimento etnográfico das pesquisadoras responsáveis pela coleta de dados. Emergiram três categorias: "O cuidado durante a experiência cirúrgica: enfermagem em ação", "Cuidado Perioperatório: dispositivos possíveis no cotidiano" e "Percepção dos Enfermeiros frente ao cuidado no centro cirúrgico", conforme segue quadros descritivos.

O Quadro 1 traz as unidades de registros que compuseram a categoria "O cuidado durante a experiência cirúrgica: enfermagem em ação". 
Research, Society and Development, v. 10, n. 11, e105101119331, 2021

(CC BY 4.0) | ISSN 2525-3409 | DOI: http://dx.doi.org/10.33448/rsd-v10i11.19331

Quadro 1 - Categorização sobre ações das enfermeiras na experiência cirúrgica.

\begin{tabular}{|c|c|c|}
\hline Categoria & Unidade de Registro & Falas dos sujeitos \\
\hline \multirow[t]{5}{*}{$\begin{array}{l}\text { o cuidado } \\
\text { durante a } \\
\text { experiência } \\
\text { cirúrgica: } \\
\text { enfermagem } \\
\text { em ação }\end{array}$} & \multirow{5}{*}{$\begin{array}{l}\text { Cuidado; Demanda; } \\
\text { Fazem o melhor; Fala de } \\
\text { funcionários; } \\
\text { Melhorar a situação do paciente; } \\
\text { Tempo não permite; Cuidado } \\
\text { fundamental; } \\
\text { Sistematização da assistência; } \\
\text { Burocracia; Trabalho técnico; } \\
\text { Diminuir riscos; } \\
\text { Estratégias Segurança. }\end{array}$} & $\begin{array}{l}\text { ENF1: } O \text { cuidado de nós enfermeiros durante esse período é vital e de extrema } \\
\text { importância, é através dele que eu tenho em partes certa gratificação, mesmo sendo } \\
\text { sozinha para entender os três setores faço da melhor forma que consigo, a demanda é } \\
\text { muito alta, mais procuro estar presente e sanar as dúvidas e aflições daqueles que estão } \\
\text { vulneráveis a nós. }\end{array}$ \\
\hline & & $\begin{array}{l}\text { ENF2: Quando se fala em cuidado esse setor que amo de paixão deixa a desejar, a parte } \\
\text { mais importante que é o cuidado que devemos oferecer as pessoas mais vulneráveis quase } \\
\text { não acontece. Tenho consciência disso mais é muita demanda de trabalho, chega a ser } \\
\text { desumano com a minha equipe. }\end{array}$ \\
\hline & & $\begin{array}{l}\text { ENF3: Minha perspectiva sempre foi muito boa em relação ao cuidado prestado aqui, } \\
\text { pensava que poderia estar presente recebendo o paciente e fazendo o que seria } \\
\text { necessário para tornar aquele momento menos obscuro a ele, mais a demanda não me } \\
\text { permite infelizmente. Na minha percepção em relação a essa pergunta existe vários vieses } \\
\text { o meu cuidado a minha atenção e muito menor que as do técnico mesmo sendo } \\
\text { assistencial a nossa função acaba sendo quase que totalmente gerencial, ficando o } \\
\text { cuidado direto ao paciente com os demais da equipe. Nesse sentido acredito que todos os } \\
\text { enfermeiros e de toda a equipe deveriam ser totalmente modificados, vejo um cuidado } \\
\text { mecanizado totalmente e somente medicamentoso. }\end{array}$ \\
\hline & & $\begin{array}{l}\text { ENF4: } O \text { cuidado do enfermeiro em centro cirúrgico para mim é de fundamental } \\
\text { importância, pois o mesmo favorece a avaliação das condiçôes de saúde e o } \\
\text { planejamento da assistência prestada ao paciente podendo analisar as condiçôes físicas, } \\
\text { psicológicas, medos, dúvidas. Representando o início da sistematização da assistência de } \\
\text { enfermagem, sendo que se o enfermeiro for qualificado pode com isso identificar as } \\
\text { necessidades dos pacientes, possibilitando a prevenção de complicações, bem como a } \\
\text { detecção precoce de intercorrências que podem acontecer no perioperatório. }\end{array}$ \\
\hline & & $\begin{array}{l}\text { ENF4: Na minha percepção nós enfermeiros nos envolvemos muito na parte } \\
\text { burocráticas/ administrativa no C.C, deixando muitas vezes em prestar um atendimento } \\
\text { humanizado/qualificado ao paciente e seus familiares. Também na minha opinião } \\
\text { desenvolvemos um trabalho muito técnico não dando atençáo as reais necessidades dos } \\
\text { pacientes exigidas no momento. O cuidado em centro cirúrgico deveria cada vez mais } \\
\text { criar estratégias e dispositivos para diminuir os riscos para os pacientes e aumentar os } \\
\text { padrões de qualidade da assistência dos serviços de saúde. Vejo a importância de } \\
\text { implementação de ações desenvolvidas pela OMS como cirurgias seguras salvam vidas, } \\
\text { dispondo de estratégias para diminuir os riscos em C.C para o paciente e a equipe que } \\
\text { presta o atendimento. }\end{array}$ \\
\hline
\end{tabular}

Fonte: Autores (2021).

O Quadro 2 traz as unidades de registros que compuseram a categoria "Cuidado Perioperatório: dispositivos possíveis no cotidiano". 
Quadro 2 - Categorização dos dispositivos usados no cuidado.

\begin{tabular}{|c|c|c|}
\hline Categoria & Unidade de Registro & Falas dos sujeitos \\
\hline \multirow[t]{4}{*}{$\begin{array}{l}\text { Cuidado } \\
\text { Perioperatóri } \\
\text { o: } \\
\text { dispositivos } \\
\text { possíveis no } \\
\text { cotidiano }\end{array}$} & \multirow{4}{*}{$\begin{array}{l}\text { Dispositivos Cuidado; } \\
\text { Demanda em excesso; } \\
\text { Uso do checklist; } \\
\text { Consulta enfermagem; } \\
\text { Atuação gerencial; } \\
\text { Trabalho mecanizado; } \\
\text { Falta de acolhimento; } \\
\text { Falta recursos humanos; } \\
\text { Criar estratégias e dispositivos; } \\
\text { Diminuir riscos; Aumentar } \\
\text { padrão de qualidade. }\end{array}$} & $\begin{array}{l}\text { ENF1: Existe somente o checklist realizado na sala de recepção da paciente, função } \\
\text { essa realizada pelos técnicos de enfermagem, seria minha função fazer a consulta de } \\
\text { enfermagem o acolhimento dos pacientes mais infelizmente não faço porque a demanda } \\
\text { gerencial tomo todo o meu tempo, e nossos pacientes ficam sem nosso cuidado. E o } \\
\text { pouco de atenção que recebem é totalmente mecanizado. }\end{array}$ \\
\hline & & $\begin{array}{l}\text { ENF2: Nós não realizamos a consulta de enfermagem nem o acolhimento, a única coisa } \\
\text { existente é o checklist e mesmo assim extremamente básico, já é um avanço, porem } \\
\text { totalmente falho pois é extremamente técnico não se vê o paciente e sim a cirurgia que ele } \\
\text { irá fazer, função que seria minha é delegada aos técnicos de enfermagem, não existe } \\
\text { acolhimento humanizado, não se sana os anseios e dúvidas do paciente não se tem } \\
\text { recursos humanos para prestar o cuidado ao paciente, fizemos da melhor forma que } \\
\text { podemos com o que temos. }\end{array}$ \\
\hline & & $\begin{array}{l}\text { ENF3: Totalmente falho fizemos o máximo que podemos, as vezes nem o checklist se faz } \\
\text { nas emergências não conseguimos fazer, falta de recursos humanos de sobrecarga de } \\
\text { funções e de trabalho faz a nossa assistência ficar na mão dos técnicos da equipe os quais } \\
\text { também trabalham sobrecarregados }\end{array}$ \\
\hline & & $\begin{array}{l}\text { ENF4: O cuidado em centro cirúrgico deveria cada vez mais criar estratégias e } \\
\text { dispositivos para diminuir os riscos para os pacientes e aumentar os padrões de } \\
\text { qualidade da assistência dos serviços de saúde. Vejo a importância de implementação de } \\
\text { ações desenvolvidas pela OMS como cirurgias seguras salvam vidas, dispondo de } \\
\text { estratégias para diminuir os riscos em C.C para o paciente e a equipe que presta o } \\
\text { atendimento, dentre estas açôes posso citar o checklist que vem consolidar a segurança } \\
\text { dos procedimentos cirúrgicos. }\end{array}$ \\
\hline
\end{tabular}

Fonte: Autores (2021).

O Quadro 3 traz as unidades de registros que compuseram a categoria "Percepção dos Enfermeiros frente ao cuidado no centro cirúrgico".

Quadro 3 - Categorização das percepções do cuidado.

\begin{tabular}{|c|c|c|}
\hline Categoria & Unidade de Registro & Falas dos sujeitos \\
\hline \multirow[t]{4}{*}{$\begin{array}{l}\text { Percepção dos } \\
\text { Enfermeiros } \\
\text { frente ao } \\
\text { cuidado no } \\
\text { centro } \\
\text { cirúrgico }\end{array}$} & \multirow{4}{*}{$\begin{array}{l}\text { Efetividade; Resolutivo; } \\
\text { Delegar funções; } \\
\text { Equipe comprometida; } \\
\text { Sensação de impotência; } \\
\text { Demanda excessiva; } \\
\text { Falta de enfermeiros; } \\
\text { Sistema hierarquizado; } \\
\text { Atividades gerenciais elevadas; } \\
\text { Assistência falha; } \\
\text { Falta de RH; } \\
\text { Cobrança; } \\
\text { Rotina; } \\
\text { Cotidiano; } \\
\text { Esquecemos que há um ser } \\
\text { humano; } \\
\text { Ausência. }\end{array}$} & $\begin{array}{l}\text { ENF1: Quando ele acontece sim é efetivo e resolutivo, busco sempre estar presente para } \\
\text { dar o mínimo de atenção que eles necessitam, mesmo tendo que delegar certas funções } \\
\text { que cabem a mim, tenho uma equipe na qual é muito comprometida com o fazer certo, e } \\
\text { isso me dá um certo conforto. Mais a sensação de se sentir impotente principalmente em } \\
\text { dias caóticos é inevitável. }\end{array}$ \\
\hline & & $\begin{array}{l}\text { ENF2: Muita demanda e responsabilidade a mim, tenho que responder por } 3 \text { setores no } \\
\text { mesmo tempo, na grande maioria dos dias agenda superlotada, fora a demanda de } \\
\text { emergência. O problema é a falta de mais enfermeiros para priorizar o atendimento. As } \\
\text { vezes sinto me extremamente impotente. }\end{array}$ \\
\hline & & $\begin{array}{l}\text { ENF3: Sempre que acontece o cuidado ele é efetivo, mais isso acontece muito raramente, } \\
\text { querem produção e tenho que entregar isso a eles. Trabalhamos num sistema totalmente } \\
\text { hierarquizado, somos responsáveis por uma parte gerencial e burocrática muito grande o } \\
\text { que nos limitas a prestar a assistência direta ao paciente, nossa assistência é totalmente } \\
\text { falha, tenho plena consciência disso mais sozinha não se consegue dar conta de tudo, } \\
\text { falta recursos humanos em todos os sentidos, mais junto com isso vem a cobrança, e a } \\
\text { rotina do cotidiano faz nos esquecermos que ali existe um ser humano e não o paciente da } \\
\text { apendicite, da fratura de perna. }\end{array}$ \\
\hline & & $\begin{array}{l}\text { ENF4: Sempre resolvemos as intercorrências que acontece, porém é muito trabalho, } \\
\text { sozinha não se consegue dar conta. É muitos profissionais inseridos no setor, todos } \\
\text { querendo aprender, isso de certa forma interfere no cuidado que deveríamos prestar. } \\
\text { Somos falhos nisso deixamos de fazer o que compete a nós para de certa forma dar } \\
\text { oportunidade a quem precisa a prender, isso tem os dois lados. Um bom porque o } \\
\text { paciente está recebendo o que precisa. Por outro lado, fica a nossa ausência. }\end{array}$ \\
\hline
\end{tabular}

Fonte: Autores (2021). 


\section{Discussão}

Ao iniciar as discussões é importante voltar o olhar para o cotidiano vivido no centro cirúrgico e as ações de cuidado desenvolvidas neste espaço. Os enfermeiros atuam diariamente na gestão do cuidado e dividem seu tempo entre organizar sua equipe para assistir o paciente durante a sua experiência cirúrgica e atender a demanda de atividades administrativas. Assim, vivenciam a perspectiva também explorada em outro estudo, de que o compartilhamento das ações no cotidiano de trabalho permite a reconfiguração de novas estratégias com intuito de cuidado qualificado. No entanto, não compartilhar a dupla ação assistencial-gerencial e por inúmeras razões, essa dualidade é demarcada por um distanciamento no cuidar humanizado desses enfermeiros, levando a muitas indagações (Christoforo \& Carvalho 2008).

A experiência pela qual o paciente cirúrgico é submetido durante todo o período perioperatório é singular para este e para a sua família, sendo relevante quando se fala de sucesso cirúrgico. Nesse sentido, o bem-estar do paciente deve constituir o principal objetivo dos profissionais que prestam assistência no período perioperatório (Moser et al, 2018).

\section{O cuidado durante a experiência cirúrgica: enfermeira em ação}

Todos os aspectos vivenciados pelos pacientes durante a experiência cirúrgica devem ser visualizados e minimizados pela enfermeira e pela equipe de enfermagem, considerando a importância do paciente no processo. Durante a realização do estudo foi possível observar que os participantes da pesquisa têm o entendimento da relevância de sua participação na experiência cirúrgica dos pacientes, sendo esclarecidas e proativas no desenvolvimento de sua função, buscando sempre atender as necessidades dos pacientes sejam eles eletivos ou urgências/emergências.

Os momentos que antecedem o ato anestésico é o período onde o medo, insegurança e a instabilidade emocional (Rocha et al, 2016) tomam conta do paciente, e aliado a essas condições emocionais, o ambiente estranho e frigido, pessoas estranhas a ele e diversos equipamentos o que acabam se tornando fatores negativos que podem comprometer a recuperação no pós-operatório.

Identificou-se, entretanto, no decorrer das observações de campo que existe uma ausência de enfermeiros desempenhando funções de cuidado e atenção aos pacientes, sendo que os mesmos atuam integralmente nas questões administrativas. Destaca-se que o bloco cirúrgico é organização complexa que vai muito além da estrutura, equipamentos ou aparelhos. Entende-se então que ao planejar e desenvolver ações junto ao paciente cirúrgico, independentemente do período operatório que se encontra, o enfermeiro deve estar aberto, disposto e disponível para perceber as necessidades e as carências apresentadas pelos pacientes, entendendo sua individualidade e atendendo-os em sua integralidade, articulando as ações com os demais membros da equipe estimulando o trabalho interprofissional (Amthauer \& Souza, 2014).

As condutas dos enfermeiros refletem diretamente no paciente, seja de forma negativa ou positiva. A conduta do Enfermeiro (a) possibilita a minimização do estresse, da ansiedade e do desgaste bio/psico/emocional/espiritual que o paciente vivencia no processo cirúrgico, proporciona segurança, tranquilidade se torna a referência. Sendo a doença o fator desencadeante da fragilidade dos pacientes e tendo este que se submeter a uma cirurgia para tratamento, importa ao cuidador em ambiente cirúrgico considerar igualmente os desgastes físico e psicológico que afloram sensivelmente no ser humano que enfrenta um processo cirúrgico, independentemente da fase perioperatória vivida (Dorneles et al, 2010).

\section{Cuidado perioperatório: dispositivos possíveis no cotidiano}

A equipe de enfermagem busca trabalhar e prestar assistência individualizada ao paciente, estabelecendo e desenvolvendo estratégias de ação no cuidado com o paciente tendo em vista as particularidades de cada procedimento cirúrgico ao qual o paciente será exposto, baseando esse cuidado em conhecimentos especializados, de forma crítica e reflexiva em sua integralidade (Koch et al, 2018). 
Observou-se durante o estudo, que tais aspectos relatados não se fazem presentes em sua integralidade, pois, durante a coleta de dados não foi possível observar a presença do enfermeiro, exercendo o cuidar diretamente com o paciente, contemplando a individualidade do paciente, na tentativa de minimização dos anseios, do estresse durante a permanência do paciente no setor.

A assistência ao paciente cirúrgico em todas as etapas perioperatória, implica em várias ações que os profissionais precisam observar, frente a segurança do paciente. O cuidado no pré, trans e pós-operatório determina a qualidade da assistência prestada e a recuperação do paciente submetido ao procedimento cirúrgico (Silva et al. 2019). Na segurança do paciente e na qualidade da assistência perioperatória, o enfermeiro é um profissional com potencial para desenhar processos de melhoria contínua da assistência, atuando no planejamento e criação de estratégias com vistas a diminuição de erros por parte de toda a equipe, buscando as boas práticas na assistência ao paciente (Guitierres et al, 2018).

\section{Percepção dos enfermeiros frente ao cuidado no centro cirúrgico}

Ao considerar os avanços técnicos e científicos na área perioperatória, a qual propicia de maneira abrangente infinitas possibilidades, no entanto, a ação humana ainda é fundamental durante o tratamento cirúrgico. O cuidado que a enfermagem propicia ao paciente e o vínculo criado através afetividade e confiança, fazem a diferença na ação de cuidar efetiva.

A ausência de capacitação técnica do enfermeiro em CC foi perceptível, existindo também dificuldades na implementação de uma assistência qualificada pelos enfermeiros no setor, justificadas pelas características diferenciais e específicas de cada paciente cirúrgico ou mesmo a rotinas adotadas na instituição (Gomes et al, 2014).

Dentre todas as falas dos enfermeiros participantes da pesquisa a principal dificuldade encontrada pelos enfermeiros referiu-se à ausência de êxito nas atividades gerenciais e assistenciais, de forma concomitante. Referindo a falta com relação ao dimensionamento e alocação de pessoal e também a sobrecarga de atividades administrativas, o que diminui o tempo do enfermeiro no processo de cuidado. Assim, os enfermeiros atribuíram a dificuldade na realização da gerência do cuidado à grande quantidade de atribuições gerenciais (Gouveia et al, 2020).

Observou-se também o sentimento de impotência e incapacidade de operacionalizar as suas atribuições gerenciais e do cuidado. Falam sobre o conflito de papéis no que tange o cuidado, eles demonstram que os enfermeiros em suas atribuições enfrentam um conflito entre o desejo de prestar cuidados e as reais cobranças da instituição: as atividades gerenciais. Observaram que os enfermeiros têm dificuldades em gerenciar o cuidado (Reis et al, 2019). Nesse sentido, os enfermeiros do centro cirúrgico afastam-se do cuidado direto ao paciente dando prioridade a suprimento de materiais e equipamentos para a unidade, e cuidados técnicos (Ribeiro, 2018).

É notável a exigência nesses cenários, de prática de um cuidado diferenciado, especialmente no centro cirúrgico por suas particularidades, sendo nesse sentido necessária a aplicação de um maior conhecimento por parte do enfermeiro. O cuidado prestado pelo enfermeiro em centro cirúrgico resulta da união de saberes acerca do manuseio do maquinário, da fisiopatologia da doença, da semiologia, dos elementos fundamentais e da subjetividade do cuidado (Ribeiro et al, 2017).

As limitações do estudo estão relacionadas ao número restrito de participantes e pode refletir apenas a realidade local onde a pesquisa foi desenvolvida, no entanto, o objetivo da proposta do estudo foi alcançada, visto que permitiu evidenciar a compreensão dos enfermeiros, atuantes no CC, sobre sua prática.

\section{Considerações Finais}

Evidenciou-se através desta pesquisa que, se faz necessário, o enfermeiro coordenador e assistencial, sendo este último com atuação direta na oferta do cuidado ao paciente junto à equipe multidisciplinar. Destacou-se a sobrecarga de 
trabalho ao qual toda a equipe de enfermagem está exposta, sendo esse o principal fator que restringe a atuação do enfermeiro junto ao paciente na oferta do cuidado.

Verificou-se que existem fragilidades dos profissionais das distintas equipes no que concerne à dinâmica do cuidado ofertado ao paciente a partir do momento que este adentra ao centro cirúrgico. Percebeu-se que existem enfermeiros que referem querer mudar a realidade, no entanto, a alta demanda, sobrecarga de trabalho e falta de recursos humanos restringe tal mudanças, e a implementação da assistência ao paciente submetido a procedimento cirúrgico.

\section{Referências}

Amthauer, C. \& Souza, T. P. (2014). The nurse's care practice to the surgical patient: an academic experience. Revista da Universidade Vale do Rio Verde, 2(12), 507-512.

Bardin, L. (2016). Análise de Conteúdo.

Christoforo, B. E. B. \& Carvalho, D. S. (2008). Nursing care performed to the surgical patient in the preoperative period. Revista Escola de Enfermagem USP, 43(1), 14-22.

Dorneles, C. G., Baggio, M. A., Nascimento, K. C., Lorenzini, E A. (2010). Cuidado perioperatório sob o olhar do cliente cirúrgico. Rev Rene, 3(11), 132-142.

Gomes, L. C., Dutra, K. E. \& Pereira, A. L. S. (2014). The nurse in the management of the operating room. Revista Eletrônica da Faculdade Metodista Granbery, 16(1), 1-21.

Gouveia, L. H. A., Ribeiro, V. F. \& Carvalho, R. (2020). Satisfação profissional de enfermeiros que atuam no bloco cirúrgico de um hospital de excelência. Rev Sobecc, 25(1), 33-41.

Gutierres, L. S., Santos, J. L. G., Peiter, C. C., Menegon, F. H. A., Sebold, L. F. \& Erdmann, A. L. (2018). Good practices for patient safety in the operating room: nurses' recommendations. Rev Bras Enferm, 71(Supl 6), 2775-82.

Johnstone, J. (2020). How to provide preoperative care to patients. Nursing Standard, 35(12), 72-6.

Klein, S., Aguiar, D. C. M., Moser, G. A. S., Hanauer, M. C. \& Oliveira, S. R. (2019). Segurança do paciente no contexto da recuperação pós-anestésica: um estudo convergente assistencial. Rev. Sobecc, 24(3), 146-153.

Koch, T. M., Aguiar, D. C. M., Moser, G. A. S., Hanauer, M. C., Oliveira, D. \& Maier, S. R. O. (2018). Surgical anesthetic moment: moving between nurses' knowledge and nursing care. Rev. Sobecc, 23(1), 7-13.

Minayo, M. C. S. \& Costa, A. P. (2018). Fundamentos teóricos das técnicas de investigação qualitativa. Revista Lusófona de Educação, 40(1),139-53.

Moser, D. C., Silva, G. A., Maier, S. R. O., Barbosa, L. C. \& Silva, T. G. (2018). Nursing care systematization: the nurses’ perception. Rev Fun Care Online, 10(4), 998-1007.

Reis, D. O. N. S., Meneses, R. O., Pinto, C. M. I., Silva, M. V. G. \& Teixeira, N. F. (2019). Indicadores gerenciais do mapa cirúrgico de um hospital universitário. Rev Sobecc, 24(4), 217-223.

Ribeiro, M. Peniche, A. C. G. \& Silva, S. (2017). Complications in the anesthetic recovery room, risk factors and nursing interventions: an integrative review. Rev Sobecc, 22(4), 218-229.

Ribeiro, M. O. S. (2018). Unveiling being a woman who experiences the preoperative hysterectomy: contributions to health care. Dissertação (Mestrado) Curso de Enfermagem, Universidade Federal de Juiz de Fora. 2018;83.

Rocha, N. M. C., Silva, F. A. A., Rocha, R. C., Rocha, J. C. \& Cabral, C. V. S. (2016). Sentimentos vivenciados por pacientes no pré-operatório. R. Interd., $9(2), 178-186$.

Santos, R., Silva, I. D. L. S., Pereira, V. A. P., Silva, M. B. S. \& Araujo, L. C. N. A. (2018). The role of the nurse in the operating room. Gep News, 2(2), 9-15.

Silva, F. R. S., Oliveira, I. R. D., Aciocle, T. H. M., Figueiredo, R. A., Silva, H. C. \& Almeida Jr, J. J. (2017). The nursing work process in the operating room: a brief account of what the literature brings. Convibra, 1(1),1-9.

Silva, H. R., Mendonça, W. A. V., Gonçalves, R. A., Sampaio, C. E. P. \& Marta, C. B. (2019). Percepção da equipe de enfermagem quanto as contribuições da utilização do checklist de cirurgia segura. Revista Enfermagem Atual In Derme, 87(25),1-8.

Sobecc - Sociedade Brasileira de Enfermeiros de Centro Cirúrgico, Recuperação Anestésica e Centro de Material e Esterilização. Practice Guidelines in Surgical Nursing and Health Product Processing-SOBECC. (7a ed.), SOBECC. 2017. 